\title{
High magnetoresistivity of a system of weakly coupled superconducting chains
}

\author{
K. B. Efetov $\left({ }^{*}\right)$ \\ Laboratoire de Physique des Solides (**), Bâtiment 510, Université de Paris-Sud, \\ Centre d'Orsay, 91405 Orsay, France
}

(Reçu le 11 janvier 1983, accepté le 25 mars 1983)

Résumé. - Nous calculons la résistivité d'un ensemble de chaînes supraconductrices faiblement couplées. Nous trouvons qu'un champ magnétique extérieur peut influencer considérablement cette résistivité.

Abstract. - The resistivity of a system of weakly coupled superconducting chains with breaks is calculated. It is shown that an external magnetic field can influence considerably this resistivity.

A very strong dependence of conductivity of the quasi-one-dimensional compounds (TMTSF $)_{2} \mathrm{PF}_{6}$ on the external magnetic field has been discovered recently [1]. This dependence was observed at temperatures $T$ which were higher than the temperature $T_{\mathrm{c}}$ of superconducting transition. The organic compound (TMTSF) ${ }_{2} \mathrm{PF}_{6}$ is a very anisotropic material with three different anisotropy constants. The simplest form of the electron energy of such a metal $\varepsilon(p)$ is

$$
\varepsilon(p)=\sum_{i=a, b, c} t_{i}\left(1-\cos p_{i} d_{i}\right)-\varepsilon_{0}
$$

where $a, b, c$, stand for different axes in the metal. In (TMTSF) ${ }_{2} \mathrm{PF}_{6}$ all $t_{i}$ are different. The value $t_{c}$ is very small [2]. It enables us to consider the compounds as a system of practically decoupled conducting planes. Furthermore, the following inequality is fulfilled

$$
t_{b} \ll t_{a}
$$

although $t_{b}$ is large enough so that

$$
t_{b} \gg t_{c}
$$

Inequality (2) allows each plane to be considered as a system of chains.

(*) Permanent address : L. D. Landau Institute for Theoretical Physics, Moscow, U.S.S.R.

(**) Laboratoire associé au CNRS. 
Experimentally the influence of the magnetic field is strongest when it is applied along the $c$ direction.

The strong dependence of conductivity on the magnetic field was attributed [2] to the existence of one-dimensional superconductivity on each chain. These chains can be considered as independent provided the interchain coupling is small enough and the temperature is low. However, the explanation in terms of one-dimensional superconductivity encounters two difficulties :

1) It is not clear why one-dimensional superconductivity can exist in (TMTSF) ${ }_{2} \mathrm{PF}_{6}$ because the necessary condition for this is

$$
t_{b} \lesssim T_{\text {co }}
$$

where $T_{\mathrm{co}}$ is the $1 \mathfrak{D}$ transition temperature.

According to the conjecture of reference [2], $T_{\text {co }} \sim 10 \div 20 \mathrm{~K}$. Apparently the condition (4) is not fulfilled in (TMTSF) ${ }_{2} \mathrm{PF}_{6}$.

2) If the chains are weakly coupled $t_{b} \ll T_{\text {co }}$ a temperature $T_{c} \ll T_{\text {co }}$ exists where the system becomes a real 3D superconductor. In the range of temperatures $T_{\mathrm{c}}<T<T_{\mathrm{co}}$ a description in terms of separate chains with the developed superconducting gap $|\Delta|$ is valid. However in this range the magnetic field does not seem at first glance to be important because it practically does not induce diamagnetic currents (only the case $\left(T-T_{c}\right) / T_{c} \ll 1$ is an exception).

Nevertheless the possibility of one dimensional superconductivity can not be rejected by these two arguments. The superconducting pairing can be due to an interaction between small open parts of the Fermi surface. All other parts may be closed by a dielectric gap. Such a situation would lead to one-dimensional superconductivity. Besides the value $t_{b}$ has not been determined unambiguously. Defects can diminish the anisotropy observed experimentally. So, objection 1 requires a more detailed study.

Another way to check the concept of one-dimensional superconductivity is to calculate the conductivity for a model of separate superconducting chains and compare the results with experimental data. This way seems to be simpler because it does not require any clear understanding of the origin of the superconductivity.

Below we consider a model of superconducting weakly coupled chains which is sensitive to the applied magnetic field. We suppose that these chains constitute a two-dimensional array. The possibility of an electron flowing from one conducting plane to another will be neglected according to the experimental situation. In this model an interaction between chains is extremely important because each chain can have breaks. An electron flow in the longitudinal direction is possible if electrons tunnel through the breaks or hop from one chain to another. Only the latter process is sensitive to the applied magnetic field. So, we consider in detail the case of tunneling from one chain to another.

Let us assume that the average distance $l$ between the breaks is much larger than all microscopic lengths in the system and, in particular, much larger than the amplitude of the hops $T_{i j}$ of electrons from one chain to another. In this limit we neglect a mutual influence of the breaks. If the probabilities of tunnelling through the breaks and from one chain to another are small these two mechanisms of transport can be considered independently. If the intrinsic longitudinal conductivity $\sigma_{\|}$between breaks is much larger than the intrinsic transverse conductivity $\sigma_{\perp}$ we obtain after a basic examination the following expression for the effective parallel conductivity

$$
\sigma_{\text {eff }}=c l^{2} / d^{2} \cdot \sigma_{\perp},
$$

where $c$ is a numerical factor depending on the distribution of breaks.

One factor $l / d_{b}$ in equation (5) comes from the proportionality of the probability of tunnelling to the length $l$ between the breaks. This factor is squared because the average transport velocity 
along the chains is also proportional to $l$. It follows from equation (5) that the longitudinal conductivity $\sigma_{\text {eff }}$ measured experimentally may depend on the intrinsic transversal conductivity $\sigma_{\perp}$.

We calculate below the superconducting fluctuation contribution to the transverse conductivity. In the considered range of temperatures $T_{\mathrm{c}}<T<T_{\mathrm{co}}$, only phase fluctuations are relevant. The effective Hamiltonian $H$ describing these fluctuations can be written as

$$
\hat{H}=\frac{1}{2} \sum_{i} \int\left(\hat{\rho}_{i}^{2} K^{-1}+K v^{2}\left(\frac{\partial \hat{\phi}}{\partial x}\right)-\sum_{j}^{\prime} J_{i j} \cos \left(2 \hat{\phi}_{i}(x)-2 \hat{\phi}_{j}(x)-\frac{2 e}{c} A_{b} d_{b}\right)\right) \mathrm{d} x,
$$

where $A_{b}$ is the $b$-component of the vector-potential. The Hamiltonian (6) is the direct generalization of the phase Hamiltonian proposed in references [3, 4]. Integration is performed over all pieces of the chains.

In equation (6) $\hat{\rho}_{i}$ and $\hat{\phi}_{i}$ are the density and the phase operators of the $i$ th chain respectively. These operators obey the following commutation relation

$$
\left[\hat{\rho}_{i}(x), \varphi_{j}\left(x^{\prime}\right)\right]=-i \delta_{i j} \delta\left(x-x^{\prime}\right) .
$$

The quantity $K$ represents compressibility and $v$ stands for the velocity of sound.

The third term in equation (6) is the usual Josephson coupling. The energy $J_{i j}$ is proportional to $t_{b}^{2}$. In the simplest case only the interaction of nearest neighbours $J$ is taken into account. The expression (6) for the free energy functions enables the following formula for the transverse current density $j$ to be written down immediatly

$$
j=2 e J\left\langle\sin \left(2 \phi_{i}-2 \phi_{i+1}-2 e A_{b} d_{b}\right)\right\rangle .
$$

In equation (8) the angular brackets stand for the averaging with the functional (6).

Suppose we want to calculate the transverse conductivity in an external magnetic field $H$ directed along the axis $C$. Then we may write the vector potential $A_{b}$ in equation (8) as

$$
A_{b}=H x+\tilde{A}(t)
$$

where $\tilde{A}(t)$ is a weak potential depending on time. Expanding in $\tilde{A}$ and $J$ in equations (6), (8) and keeping only the lowest non vanishing terms with respect to these variables we find after simple calculations

$$
j=Q(\omega) \tilde{A}(\omega)
$$

The response $Q(\omega)$ is the result of analytical continuation from Matsubara frequencies $i \omega_{\mathrm{n}} \rightarrow$ $\omega+i \delta$ of the function $Q\left(i \omega_{n}\right)$

$$
Q\left(i \omega_{\mathrm{n}}\right)=4 e^{2} J^{2} \int_{0}^{1 / T} \int_{-\infty}^{\infty} G_{0}^{2}(x, \tau)\left(\mathrm{e}^{i \omega_{\mathrm{n}}}-1\right) \cos (2 e H x d) \mathrm{d} x \mathrm{~d} \tau,
$$

where

$$
G_{0}(x, \tau)=\left\langle\mathrm{e}^{i \phi(x, \tau)-i \phi(0,0)}\right\rangle_{0}
$$

The symbol $\langle\cdots\rangle_{0}$ stands for the averaging with the Hamiltonian (6) taken in the limit $J \rightarrow 0$.

When deriving equations (10), (11), (12) we neglected averages of the type

$$
\left\langle\mathrm{e}^{i \phi(x, \tau)+i \phi(0,0)}\right\rangle_{0}
$$


which vanish for large $l$. The explicit expression for $G_{0}(x, \tau)$ is very well known $[3,4]$

$$
G_{0}(x, \tau)=\left(\frac{B T}{T_{\mathrm{co}}}\right)^{\alpha}\left(\sinh \pi T\left(\frac{x}{v}+i \tau\right) \cdot \sinh \pi T\left(\frac{x}{v}-i \tau\right)\right)^{-\alpha / 2},
$$

where $B$ is a number, $\alpha=2(\pi K v)^{-1}$.

Equations (11)-(13) are valid provided the effective interactions between chains are small. This condition is satisfied for high temperatures $T \gg T_{\mathrm{c}}$, where $T_{\mathrm{c}}$ is the critical temperature of the real 3D superconducting transition.

The calculation in equation (11) with the function $G_{0}(x, \tau)(13)$ is not difficult and can be performed exactly. It is convenient to deform the contour $(0,1 / T)$ when integrating over $\tau$ into a sum of the contours $(0, i \infty),(i \infty, 1 / T+i \infty)$ and $(i \infty+1 / T, 1 / T)$. After calculation of the integral and analytical continuation $i \omega_{\mathrm{n}} \rightarrow \omega+i \delta$ we obtain

$$
\begin{gathered}
Q(\omega)=F(\omega)-F(0), \\
F(\omega)=\frac{e^{2} \cdot v J^{2}}{\pi^{2} T^{2}}\left(\frac{2 T B}{T_{\mathrm{co}}}\right)^{2 \alpha} \frac{\Gamma^{-2}(\alpha)}{\sin \pi \alpha}\left|\Gamma\left(\frac{\alpha}{2}-i \frac{\omega-\tilde{H}}{4 T}\right)\right|^{2}\left|\Gamma\left(\frac{\alpha}{2}-i \cdot \frac{\omega+\tilde{H}}{4 T}\right)\right|^{2} \times \\
\times \sin \left(\frac{\pi \alpha}{2}+\frac{i(\omega-\tilde{H})}{4 T}\right) \cdot \sin \left(\frac{\pi \alpha}{2}+\frac{i(\omega+\tilde{H})}{4 T}\right),
\end{gathered}
$$

where

$$
\tilde{H}=2 e v \mathrm{~d} H
$$

$\Gamma(\alpha)$ is the Euler gamma-function

Formulae (14), (15) for $\tilde{H}=0$ have been obtained for a similar model in the reference [5].

At low frequencies $Q(\omega)$ depends linearly on the frequency. The intrinsic perpendicular conductivity $\sigma(0)$ takes the form

$$
\sigma(0)=\frac{e^{2} J^{2} \Gamma^{-2}(\alpha) v}{4 \pi^{2} T^{3}}\left(\frac{2 B T}{T_{\mathrm{co}}}\right)^{2 \alpha}\left|\Gamma\left(\frac{\alpha}{2}-\frac{i \tilde{H}}{4 T}\right)\right|^{4} .
$$

It is convenient to write the conductivity in terms of the critical temperature $T_{\mathrm{c}}$ of the real 3D superconducting transition. Considering the interaction between chains in the mean-field approximation [4] we obtain an equation for $T_{\mathrm{c}}$ in zero magnetic field

$$
1=\frac{z J}{2} \int_{0}^{1 / T_{\mathrm{c}}} \int_{-\infty}^{\infty} G_{0}(x, \tau) \mathrm{d} \tau \mathrm{d} x
$$

where $z$ is the number of nearest neighbours. In two dimensions $z=2$ (formula (17) can be applied in two dimensions because even very small interactions between planes cut logarithmic divergencies). Substituting equation (17) into equation (16) and using equation (5) we find

$$
\sigma_{\mathrm{eff}}=c(2 \pi)^{2} e^{2}\left(\frac{T_{\mathrm{c}}}{v}\right) \frac{l^{2}}{d_{b}^{2}}\left(\frac{T}{T_{\mathrm{c}}}\right)^{2 \alpha-3}\left|\Gamma\left(\frac{\alpha}{2}-\frac{i \tilde{H}}{4 T}\right)\right|^{4} f(\alpha),
$$

where

$$
f(\alpha)=\frac{\Gamma^{4}\left(\frac{\alpha}{2}\right)}{\Gamma^{2}(\alpha)} \cdot \frac{1}{\Gamma^{8}\left(\frac{\alpha}{4}\right)} \cdot \frac{\sin ^{2} \frac{\pi \alpha}{2}}{\sin ^{4} \frac{\pi \alpha}{4}} .
$$


It is seen from equation (18) that at $\tilde{H}=0$ and $T \sim T_{\mathrm{c}}$ the conductivity $\sigma_{\text {eff }}$ can be very large. If $\alpha<3 / 2$ the conductivity grows with decreasing temperature in a power law.

However in a non-zero magnetic field the conductivity begins to fall off at temperatures $T<\tilde{H}$. This assertion follows immediately from the asymptotics of the $\Gamma$-function

$$
\Gamma(a-i b) \sim \mathrm{e}^{-\pi b}, \quad b \rightarrow \infty .
$$

Let us recall that $\sigma_{\text {eff }}(18)$ describes only the magnetic field dependent contribution. Another contribution comes from the possibility of tunnelling through breaks. Besides, the one particle contribution can also exist. The resistivity $R$ which could be observed experimentally is

$$
R=\left(\sigma_{\text {eff }}+\sigma_{0}\right)^{-1}
$$

where $\sigma_{0}$ is the field independent contribution. It follows from equations (18), (19) that $R(T)$ has a minimum for finite $H$. The position of this minimum is determined by the following equation

$$
(3-2 \alpha)+2 X \frac{\mathrm{d}}{\mathrm{d} x}\left|\Gamma\left(\frac{\alpha}{2}-i x\right)\right|^{2}=0,
$$

where

$$
x=\frac{\tilde{H}}{4 T}
$$

If $\alpha$ is close to $3 / 2$ the minimum corresponds to rather high temperatures

$$
T=\frac{1}{2} \tilde{H} \frac{\left(\Gamma\left(\frac{3}{4}\right) \Gamma^{\prime \prime}\left(\frac{3}{4}\right)-\left(\Gamma^{\prime}\left(\frac{3}{4}\right)\right)^{2}\right)^{1 / 2}}{(3-2 \alpha)^{1 / 2}} .
$$

At low temperatures $\sigma_{\text {eff }}$ in equation (19) can be neglected. Therefore in the limit $T \ll \tilde{H}$ the resistivity is equal to $\sigma_{0}^{-1}$. Of course, we consider the behaviour only above the superconducting transition.

In a small magnetic field, the resistivity $R$ can decrease monotonically wich temperature because the superconducting transition is reached earlier than the minimum determined by equation (20).

The behaviour described by equations (18), (19) is in a good agreement (at least qualitative) with the experimental data for (TMTSF) ${ }_{2} \mathrm{PF}_{6}[1]$. For a more detailed comparison of the theory and the experiment it is necessary to know the values of $\alpha$ and $v$, which determine for example the position of the minimum (Eqs. (20), (15a)). Let us emphasize that this interesting dependence on the magnetic field can be seen only in kinetics. If we are not close to the 3D superconducting transition, the thermodynamics is determined by the $J=0$ limit of the Hamiltonian (6). This limit corresponds to free sound phonons. Therefore, one may conclude that the contribution from these superconducting fluctuations to $f, e$, the specific heat is proportional to $T$ because the Hamiltonian $\hat{H}(6)$ is one-dimensional.

The index $\alpha$ depends on interactions [4] and impurities [6]. So, it is quite reasonable to expect that in some materials this index can be in the interval $\frac{3}{2}<\alpha<2$. For such values of $\alpha$ the conductivity $\sigma_{\text {eff }}(18)$ decreases with decreasing temperature even at $H=0$. It corresponds to an increase of resistivity above the 3D superconducting transition.

The theory developed above is applicable only if $\alpha<2$. It is seen from equation (18) that $\sigma_{\text {eff }} \rightarrow 0$, as $\alpha \rightarrow 2$. If $\alpha>2,3 \mathrm{D}$ superconductivity can not exist and all contributions coming from long wavelength excitations described by the Hamiltonian (6) are small.

Thus, we have shown that a system of one-dimensional superconducting chains can have a large magnetoresistivity. A large magnetoresistivity can also exist in the same system of disordered metallic chains without any superconducting pairing provided the system is close to the 
Anderson mobility edge. If the electron energy is described by equation(1) with inequalities(2),(3) the mobility edge is reached when $t_{b}{ }^{\prime} \sim \tau_{\mathrm{tr}}^{-1}$, where $\tau_{\mathrm{tr}}$ is the mean free time (we do not take into account the possibility of two-dimensional localization). Due to large values of $\tau_{\mathrm{tr}}$ in (TMTSF $)_{2} \mathrm{PF}_{6}$ the mobility edge corresponds again to very small $t_{b}$. In the presence of the magnetic field $H$ directed along the $C$-axis we must add oscillating factors in terms describing tunnelling of electrons from one chain to another

$$
t_{b}\left(a_{i}^{+} a_{i+1}+a_{i+1}^{+} a_{i}\right) \rightarrow t_{b}\left[a_{i}^{+} a_{i+1} \exp \left(\frac{i e}{c} H d_{b} x\right)+a_{i+1}^{+} a_{i} \exp \left(-\frac{i e}{c} H d_{b} x\right)\right] .
$$

Due to the oscillations the magnetic field reduces the effective tunnelling amplitude thus making the system more one-dimensional and favouring the localization. A large magnetoresistivity comparable with that observed experimentally is possible only if the system is really close to the mobility edge. Then, increasing the field $H$ we come from the metallic state to the localized state where the temperature dependence of resistivity has a minimum [7]. In this model the resistivity in the localized state must go to infinity when $T \rightarrow 0$. However, the experimentally observed resistivity in rather strong magnetic fields seems to go to a finite value as $T \rightarrow 0$.

To summarize, the large magnetoresistivity above the superconducting transition is possible only if the amplitude of tunnelling $t_{b}$ is small.

The model of superconducting fluctuations seems to be more relevant to the experimental situation because the resistivity in a strong magnetic field tends to a finite value as $T \rightarrow 0$. The Hamiltonian (6) is rather general and does not require a clear understanding of the origin of superconductivity. Of course, the question why one-dimensional superconductivity with $T_{\mathrm{c}}<$ $T_{\text {co }}$ can be possible in (TMTSF) ${ }_{2} \mathrm{PF}_{6}$ still remains unclear. Apparently a considerable magnetoresistivity can be obtained when $t_{b} \sim T_{\text {co }}$. A possible one-electron interpretation of high magnetoresistivity in terms of strong variations of scattering rates in different regions of the Fermi surface was given in reference [8]. No doubt the problem of this large magnetoresistivity deserves a detailed study.

\section{Acknowledgments.}

I thank the staff of the Laboratoire de Physique des Solides, Orsay, where this work was performed, for kind hospitality.

\section{References}

[1] Jérome, D., Chemica Scripta 17 (1981) 13.

[2] Jérome, D., Schulz, H. J., Adv. Phys. 31 (1982) 299.

[3] Luther, A., Emery, V. J., Phys. Rev. Lett. 33 (1974) 589.

[4] Efetov, K. B., LARkin, A. I., Zh. Eksp. Teor. Fiz. 69 (1975) 764 ; (Sov. Phys. J.E.T.P. 42 (1975) 390).

[5] Efetov, K. B., Pis'ma Zh. Eksp. Teor. Fiz. 31 (1980) 118 ; (Sov. Phys. J.E.T.P. Lett. 31 (1980) 108).

[6] Larkin, A. I., Melnikov, V. I., Zh. Eksp. Teor. Fiz. 71 (1976) 2199 ; (Sov. Phys. J.E.T.P. 44 (1976) 1159).

[7] Gogolin, A. A., Melnikov, V. I., Rashba, E. I., Zh. Eksp. Teor. Fiz. 72 (1977) 629 ; (Sov. Phys. J.E.T.P 45 (1977) 330).

[8] Jacobsen, C. S., Mortensen, K., Weger, M., BechgaArd, K., Solid State Commun. 38 (1981) 423. 